\title{
Assessment of Contact Lens with the High Definition (HD) Property
}

\author{
RAIED A. FAGEHI, Ph.D. \\ The Department of Optometry and Vision Sciences, College of Applied Medical Sciences, King Saud University
}

\begin{abstract}
Background: Contact lenses have been in use since the 1930s. During these years, many materials have been considered with the aim of producing more advanced and biocompatible devices. The 1940 s was the era of the first modern contact lenses, with the debut of polymethyl methacrylate (PMMA). The lack of oxygen supply to the cornea was the main disadvantage of PMMA. This oxygen impermeability was solved with the development of different contact lens materials, new generation of contact lens use hydrogel and silicone to enhance oxygen permeability. Recently, the new contact lens material known as silicone hydrogel lenses has been used to produce a new lens with the HD property.
\end{abstract}

Aim of Study: The aim of this study was to assess a new high definition silicone hydrogel contact lens.

Patients and Methods: 22 subjects have been recruited from King Saud University students (male, age $21 \pm 1.5$ years). The new Balafilcon A lenses (PUREVISION 2 HD); (Bausch + Lomb, Tokyo, Japan) has been assessed and compared with another (non HD) silicone hydrogel lens Balafilcon A (PureVision, Bausch \& Lomb Inc., Rochester, NY).

The participants asked to wear each of the two lenses for one week (the lens was given randomly) and 48 hours wash out period was allowed between the lenses. Clinical tests including visual acuity (VA) and contrast sensitivity (CS) were applied to evaluate the lens performance at the end of each week. Additionally, contact lens dry eye questionnaire (CLDEQ-8) has been used to obtain the subjective feedback.

Result: The mean of CLDEQ-8 with PureVision was 8.1 and with Pure Vision 2 HD was 6 . For contrast sensitivity the mean with PureVision was 1.74 while with Pure Vision 2 HD was 1.77 . The mean of Visual Acuity was equal with the two lenses $1.00 \mathrm{log}$. Statistical tests using Wilcoxon-signed rank test showed no significant difference exist between the HD PureVision 2 and PureVision lens. For Visual Acuity ( $p=$ 0.317 ), Contrast sensitivity ( $p=0.150$ ) (Fig. 2 ) and CLDEQ$8(p=0.339)$.

Conclusion: The silicone hydrogel HD lens evaluated in this study showed good performance. However, no significant difference in clinical performance was observed between the HD lens and the non HD silicone hydrogel lens.

Correspondence to: Dr. Raied A. Fagehi, E-Mail: Rfagehi@ksu.edu.sa
Key Words: Contact lens - Silicone hydrogel - High definition (HD) - Contrast sensitivity.

\section{Introduction}

CONTACT lenses have been in use since the 1930s. Since that time many materials have been considered with the aim of producing more advanced devices. The first soft contact lenses were made from $80 \%$ 2-hydroxyethyl methacrylate (HEMA) and 20\% diethylene glycol methacrylate (DEGMA); later, pure HEMA was used, on its own, resulting in improved mechanical properties of lenses [1].

In 1979 Tanaka et al. [2] attempt to combine hydrogel with silicone. They successfully combined HEMA with a monomer (trimethylsiloxy) - methacryloxy-propylsilane (TRIS) producing a rigid lens material [2]. Tanaka also suggested inserting a polar group (hydroxyl) to modify TRIS. Copolymerising TRIS with hydroxyl (hydrophilic monomer) resulted in a material suitable for use as a soft contact lens.

The co-polymerising of TRIS with a hydroxyl group successfully enhanced the oxygen permeability but did not address the issue of limited lens movement. This issue can be explained by referring to two approaches to improving ocular compatibility which was used in the development of the first silicone hydrogel lenses.

The first approach was the incorporation of fluorine into the hydrogel structure. This improved three characteristics of contact lens: wettability, mechanical properties and oxygen permeability [3].

The second approach was the development of macromer technology. "Macromers are large monomers formed by pre-assembly of structural units 
that are designed to bestow particular properties on the final polymer" [4]. The use of macromers and their role in enhancing oxygen permeability was explained in 1991 by Robertson et al. [5]. Their construction involved the combination of hydrophilic polyethylene oxide segments with oxygen gas permeable polysiloxan units. These two approaches, TRIS modification and macromer technology, are the basis of the principle used to develop the first commercial silicone hydrogel lenses: PureVision (Bausch \& Lomb, Rochester, NY) and Focus Night \& Day (now Air Optix Night \& Day, Ciba Vision) [6]

Up until 2005, all silicone hydrogel contact lens materials either depended on modified TRIS (PureVision), alone, or together with silicone based macromers (AirOptix Night \& Day, Acuvue Advance and Acuvue Oasys).

In 2006 a new range of silicone hydrogel contact lenses was released. This family of silicone hydrogel lenses included Biofinity (Comfilcon A, CooperVision, Inc) and PremiO (Asmofilcon A, Menicon). More recently Avaira (Enfilcon A, CooperVision, Inc) has been released [7].

In 2007, Menicon Co, Ltd. (Japan) introduced PremiO lens for daily wear on a two-week replacement basis. The PremiO lens incorporates a plasma surface treatment (NanoglassTM technology) to improve comfort and reduce dryness [8].

Up until now the development in contact lenses is continuous, the contact lenses manufacturers are working hard to develop lenses more compatible and less effective on cornea and tear film stability.

As we find the sign HD (High Definition) on most of the TV screens, computers, mobiles, camera etc. The Bausch \& Lomb also produce soft contact lens known as PureVision 2 HD lens.

For the screens and cameras, the HD means high definition or more pixels leading to a brighter, clear picture. For the HD contact lenses according to the manufacturer, the HD lens improves the quality of vision by using what they call "high definition Optics".

The Pure Vision 2 HD lens is supposed to reduce spherical aberration [9], which usually affects the vision in low light. Patient with spherical aberration often suffer from impaired vision, halos or blurred vision and glare in low light [10]

The Pure Vision 2 HD was described as one of the most breathable lens available, allowing more oxygen to the cornea and they are very thin which is supposed to enhance ocular comfort [11]

The aim of this study is to investigate the performance of the new silicone hydrogel HD lens. Due to the special design of the HD lens to reduce optical aberration, clinical differences between this new HD lens and the other silicone hydrogel lens are expected to exist.

The two important goals of any contact lenses are improving vision and maintaining ocular comfort. So three common clinical tests have been applied here including visual acuity (VA) test [12] contrast sensitivity (CS) test [13] and subjective assessment with contact lens questionnaire [14-17]

\section{Patients and Methods}

22 subjects have been recruited from King Saud University students (male, age $21 \pm 1.5$ years) and asked to take part in this study. The inclusion criteria were $>18$ years old, no history of ocular or systemic diseases reported. Participants with systemic or ocular disease have been excluded from the study.

This study was Conducted at King Saud University, Riyadh, Saudi Arabia during the period from February to April 2018.

This study followed the tenets of the Declaration of Helsinki [18]. Informed consent was obtained from all individual participants included in the study.

The new Balafilcon A lenses (PUREVISION 2 HD); (Bausch + Lomb, Tokyo, Japan) performance has been assessed and compared with another (non HD) silicone hydrogel lens Balafilcon A (PureVision, Bausch \& Lomb Inc., Rochester, NY) (Table 1).

The participants are asked to wear each of the two lenses for one week (the lens was given randomly) and 48 hours wash out period was allowed between the lenses. Clinical tests including visual acuity (VA) and contrast sensitivity (CS) were applied to evaluate the lens performance at the end of each week. Additionally contact lens dry eye questionnaire (CLDEQ-8) [16] has been used to obtain the subjective feedback.

\section{Pure Vision HD Lens:}

PureVision contact lenses with high definition optics are designed to reduce spherical aberration 
across the entire power range. Aberration is a distortion acquired by a wave-front of light when it passes through an eye with irregularities.

\section{Visual acuity (VA test):}

The visual acuity test is used to determine the smallest letters you can read on a standardized chart (Snellen chart) or a card held 20 feet away.

\section{Contrast sensitivity:}

The Pelli-Robson test measures contrast sensitivity using large letters as targets (equivalent to $20 / 60$ acuity). For each group of 3 letters, contrast is decreased from left to right and from the top to the bottom of the chart. The lowest contrast at which 2 or 3 of the letters in a group can be read determines a log contrast sensitivity score. The Pelli-Robson contrast sensitivity chart is an 86$63 \mathrm{~cm}$ chart that consists of 16 triplets of $4.9 \mathrm{~cm}$ $\left(2.8^{\circ}\right.$ at $\left.1 \mathrm{~m}\right)$ letters, and it assesses contrast sensitivity (CS) at a spatial frequency of about 0.50 to 1 cycle/degree. Within each triplet, the letters have the same contrast, and the contrast in each successive triplet decreases by a factor of $0.15 \log$ units (Fig. 1).

\section{CLDEQ-8:}

The CLDEQ-8 has 5 parts, it evaluates dryness, discomfort, and "blurry vision"; frequency of "closing eyes" due to discomfort and "removing Contact Lens to relieve discomfort. It measures the impression lenses for patients by assessing each part from 0 to 4 where 0 gives the best impression and full satisfaction.

The recent International Workshop on Contact Lens Discomfort reported that the contact lens dry eye questionnaire (CLDEQ) is the only tool validated for the evaluation of contact lens induced discomfort [14].

\section{Results}

The test of normality using (KolmogorovSmirnov) showed that the data was normally distributed $(p>0.055)$. The statistical tests using Wilcoxon-signed rank test showed that no significant difference between the PureVision 2 HD and PureVision lens. For Visual Acuity $(p=0.317)$, Contrast sensitivity ( $p=0.150)$ (Fig. 2$)$ and CLDEQ-8 ( $p=$ 0.339 ) (Fig. 3).

The mean of CLDEQ-8 with PureVision was 8.1 and with Pure Vision 2 HD was 6 . For contrast sensitivity the mean with PureVision was 1.74 while with Pure Vision 2 HD was 1.77. The Visual Acuity was equal with the two lenses $1.00 \log$.
Table (1): Properties of PureVision 2 HD and PureVision lenses.

\begin{tabular}{lll}
\hline $\begin{array}{l}\text { Contact lens } \\
\text { brand name }\end{array}$ & PureVision 2 HD & \multicolumn{1}{c}{ PureVision } \\
\hline Manufacturer & $\begin{array}{l}\text { Bausch and Lomb } \\
\text { Balafilcon A }\end{array}$ & $\begin{array}{l}\text { Bausch and Lomb } \\
\text { Balafilcon A } \\
\text { Lens material }\end{array}$ \\
Modulus & 1.50 & 1.1 \\
Diameter & $14.00 \mathrm{~mm}$ & $14.2 \mathrm{~mm}$ \\
Base curve & $8.6 \mathrm{~mm}$ & $8.6 \mathrm{~mm}$ \\
Water content & $36 \%$ & $36 \%$ \\
Dk/T at lens center & 130 & 99 \\
Thickness & $0.07(-3.00 \mathrm{DS})$ & $0.09(-3.00 \mathrm{DS})$ \\
\hline
\end{tabular}

Table (2): Results of The PureVision 2 HD and PureVision lenses.

\begin{tabular}{llll}
\hline & VA $(\log )$ & CS & CLDEQ-8 \\
\hline PureVision 2 HD & 1.00 & 1.77 & 6 \\
PureVision & 1.00 & 1.74 & 8.1 \\
$p$-value & 0.317 & 0.150 & 0.339 \\
\hline
\end{tabular}

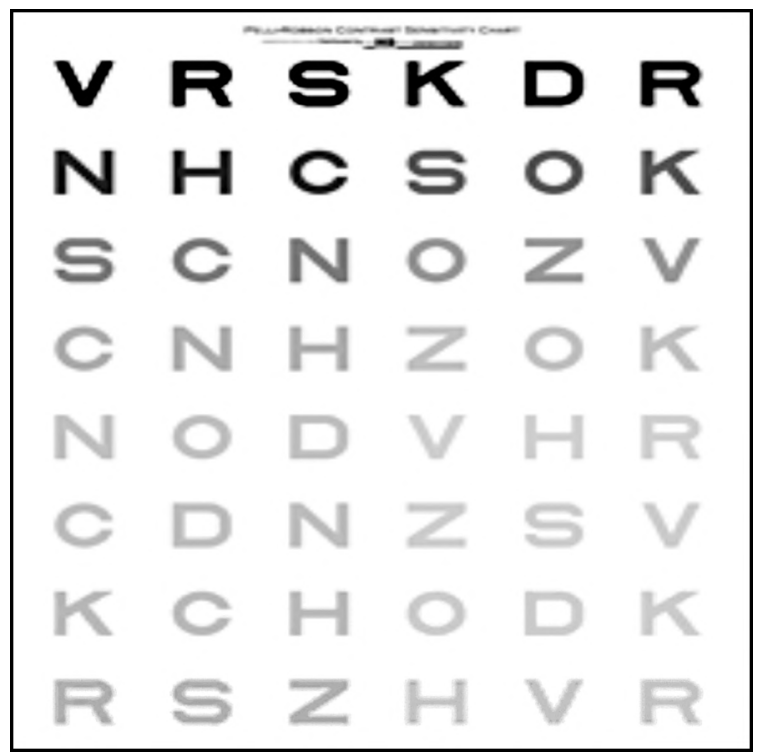

Fig. (1): Pelli Robson chart.

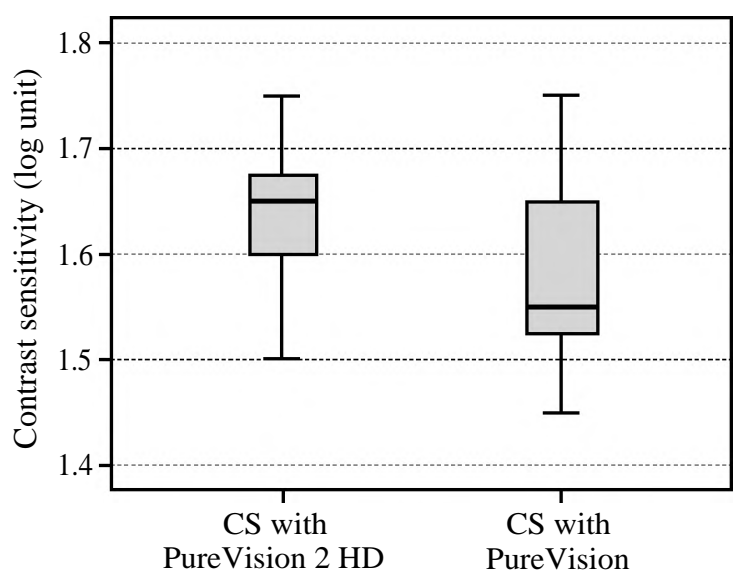

Fig. (2): Box plots showing the difference between contrast sensitivity of subjects wearing PUREVISION 2 HD and PureVision lenses. 


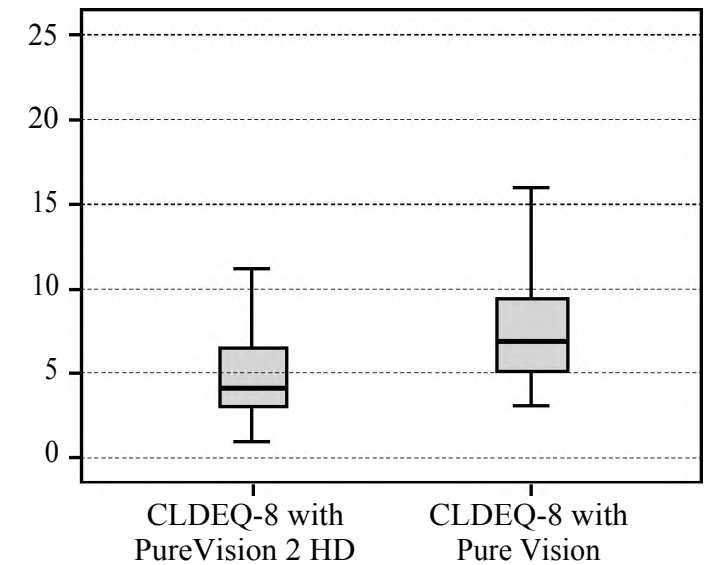

Fig. (3): Box plots showing the difference in CLDEQ-8 between subjects wearing PUREVISION 2 HD and PureVision lenses.

\section{Discussion}

This study investigates the clinical performance of new HD silicone hydrogel lens and the compares it with one of the commonly use non HD silicone hydrogel.

The two lenses studied here showed good performance in term of subjective assessment with CLDEQ-8. According to Chalmers et al. [19] the CLDEQ-8 score > 12 is diagnosed as SCL related symptoms.

The CLDEQ-8 with PureVision 2 HD was slightly better (score 6) than that with PureVision (score 8.1). This might be due to the thin thickness [9] of the Pure Vision $2 \mathrm{HD}(0.07 \mathrm{~mm})$ compared to PureVision $(0.08 \mathrm{~mm})$ [20] . However, none of the two lenses assessed showed score more than the CLDEQ-8 cutt-off value $\geq 12$ and no significant difference between the lenses was observed.

The contrast sensitivity and visual acuity evaluated of the subject when they are wearing Pure Vision $2 \mathrm{HD}$ and PureVision was not significantly different. The contrast sensitivity mean with PureVision 2 HD was $1.77 \log$ and with PureVision was $1.74 \log$.

For patients between 20 and 50 years old, CS should be $1.80 \log$ units and above; for patients less than 20 years old and older than 50 years, monocular CS should be 1.65 log units and above [21]. In our study, we observed that CS with PureVision 2 HD was slightly better and very close to the normal (1.80 log units).

No difference was observed regarding the visual acuity with the two lenses. Also, both lenses showed a good subjective feedback and good clinical performance.
The Pure Vision 2 HD lens (Balafilcon A) material is a first generation silicone hydrogel, plasma oxidation surface treated lens. PureVision 2 lenses with High Definition Optics are designed to reduce halos and glare and deliver clear vision. It offers design advances for outstanding comfort and breathability.

The similarity in subjective response observed between the two soft lenses evaluated in this study might be because both lenses have their surface treated with a layer of plasma which makes the surface hydrophilic, better tear film stability therefore more comfort to the patient [22]

These two lenses have good properties in term of water content, oxygen permeability, modulus, thickness and wettability [23-25]

These good properties might reflect on the clinical performance of the two lenses. However, a longer wearing time might be required to reach the full judgment on such lens. Additionally, objective assessment of the corneal abrasion with this new lens should be targeted in future study.

\section{Conclusion:}

The silicone hydrogel HD lens evaluated in this study showed good performance. However, no significant difference was observed when the HD lens compared with a non HD silicone hydrogel lens.

\section{Conflict of interest:}

No conflict of interest to declare.

\section{References}

1- PEARSON R.M.: A review of the limitations of the first hydrogel contact lenses. Clin. Exp. Optom., 93 (1): 15$25,2010$.

2- TANAKA K., TAKAHASHI K., KANADA M., KANOME S. and NAKAJIMA T.: Copolymer for soft contact lens, its preparation and soft contact lens made thereof, 1979.

3- KUNZLER J. and OZARK R.: Fluorosilicone hydrogels, 1994.

4- TIGHE B.: Silicone hydrogels: Structure, properties and behaviour: D. Sweeney, Editor. Oxford, ButterworthHeinemann, p. 1-27, 2004.

5- ROBERTSON J.R., SU K.C., GOLDENBERG M.S. and MUELLER K.F.: Wettable, flexible, oxygen permeable contact lens containing block copolymer polysiloxanepolyoxyalkylene backbone units and use thereof, 1991.

6- LÓPEZE-ALEMANY A., COMPAÑ V. and REFOJO M.F.: Porous structure of PurevisionTM versus Focus ${ }^{\circledR}$ Night\&DayTM and conventional hydrogel contact lenses. Journal of Biomedical Materials Research, 63 (3): 319 $25,2002$. 
7- EFRON N., MORGAN P.B., MALODONADO-CODINA C., BRENNAN N.A. and CHIRILIA T.: Contact lenses: the search for superior oxygen permeability. Biomaterials and Regenerative Medicine in Ophthalmology: Woodhead, p. 280-303, 2010 .

8- LAKKIS C. and VINCENT S.: Clinical Investigation of Asmofilcon A Silicone Hydrogel Lenses. Optom. Vis. Sci., 86 (4): 350-6, 2009.

9- KOH S., MAEDA N., HAMADA T. and NISHIDA K. Efficacy of spherical aberration correction based on contact lens power. Contact Lens and Anterior Eye, 37 (4): 273-7, 2014.

10-KOOMEN M., TOUSEY R. and SCOLNIK R.: The spherical aberration of the eye. JOSA, 39 (5): 370-6, 1949.

11- McDONNELL C.: Contact Lens Materials, 2013.

12- VA V.A.: Visual acuity, 1965.

13- PELLI D., ROBSON J., et al.: The design of a new letter chart for measuring contrast sensitivity. Clinical Vision Sciences, Citeseer, 1988.

14- FOULKS G., CHALMERS R., KEIR N., WOODS C.A., SIMPSON T., LIPPMAN R., et al.: The TFOS International Workshop on Contact Lens Discomfort: Report of the Subcommittee on Clinical Trial Design and Outcomes. Invest Ophthalmol. Vis. Sci., 54 (11): 157-83, 2013.

15- YOUNG G., CHALMERS R.L., NAPIER L., HUNT C. and KERN J.: Characterizing contact lens-related dryness symptoms in a cross-section of UK soft lens wearers Cont. Lens Anterior Eye, 34 (2): 64-70. Epub 2010/09/14, 2011.

16- CHALMERS R.L., BEGLEY C.G., MOODY K. and HICKSON-CURRAN S.B.: Contact Lens Dry Eye Questionnaire-8 (CLDEQ-8) and Opinion of Contact Lens Performance. Optom. Vis. Sci., 89 (10): 1435-42, 2012.

17- NICHOLS J.J., MITCHELL G.L., NICHOLS K.K., CHALMERS R. and BEGLEY C.: The performance of the contact lens dry eye questionnaire as a screening survey for contact lens-related dry eye. Cornea, 21 (5): 469-75. Epub 2002/06/20, 2002.

18- RICKHAM P.: Human experimentation. Code of Ethics of the World Medical Association. Declaration of Helsinki BMJ, 2 (5402): 177, 1964.

19- CHALMERS R.L., KEAY L., HICKSON-CURRAN S.B. and GLEASON W.J.: Cutoff score and responsiveness of the 8-item Contact Lens Dry Eye Questionnaire (CLDEQ$8)$ in a Large daily disposable contact lens registry. Contact Lens and Anterior Eye, 39 (5): 342-52, 2016.

20- EFRON N., BRENNAN N.A., CURRIE J.M., FITZGERALD J.P. and HUGHES M.T.: Determinants of the initial comfort of hydrogel contact lenses. Optometry \& Vision Science, 63 (10): 819-23, 1986.

21- ELLIOTT D.B., SANDERSON K. and CONKEY A.: The reliability of the Pelli-Robson contrast sensitivity chart. Ophthalmic and Physiological Optics., 10 (1): 21-4, 1990.

22- BHATTACHARYA S., DATTA A., BERG J.M. and GANGOPADHYAY S.: Studies on surface wettability of poly (dimethyl) siloxane (PDMS) and glass under oxygen-plasma treatment and correlation with bond strength. Journal of microelectromechanical systems, 14 (3): 590-7, 2005.

23- BRENNAN N.A., COLES M.L., ANG J.H.: An evaluation of silicone-hydrogel lenses worn on a daily wear basis. Clin. Exp. Optom., 89 (1): 18-25, 2006.

24- MARTIN R., de JUAN V., RODRIGUEZ G., MARTIN S. and FONSECA S.: Initial comfort of lotrafilcon A silicone hydrogel contact lenses versus etafilcon A contact lenses for extended wear. Cont. Lens Anterior Eye, 30 (1): 23-8. Epub 2006/12/21, 2007.

25- LONG B., ROBIRDS S. and GRANT T.: Six months of in-practice experience with a high Dk lotrafilcon a soft contact lens. Cont. Lens Anterior Eye, 23 (4): 112-8, 2000. 


\section{تقييم العدسات اللاصقة ذات خاصية الوضوح العالى}

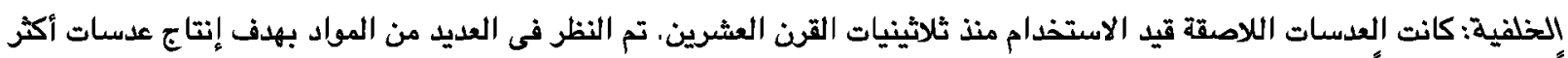

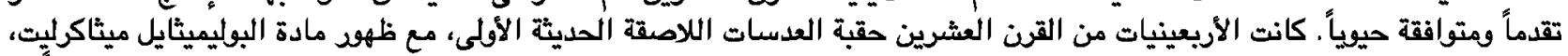

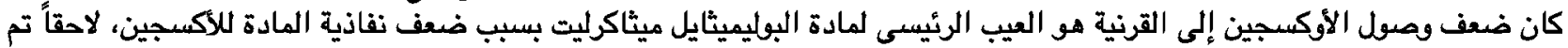

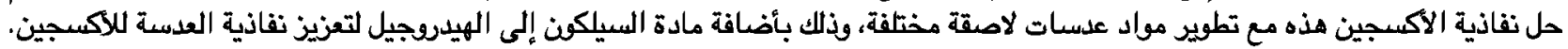

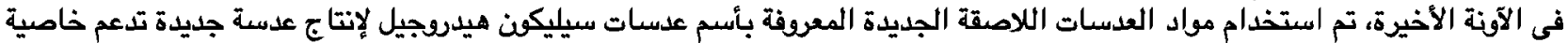
الوضوح العالى. - الإيرة.

الهدف من الدراسة: هو تقييم اداء عدسة لينة جديدة مصنوعة من مادة السيليكون هيدرجيل ؤها خاصية الوضوح العالى ومقارنتها بعدسة أخرى مصنوعة من نفس المادة ولا تملك خاصية الوضيق لهرح العالى.

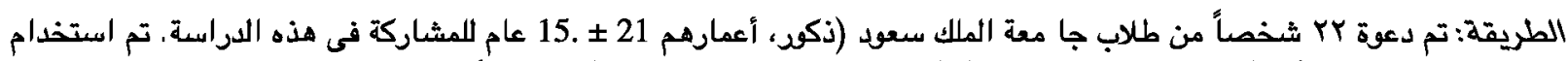

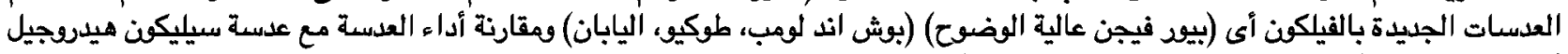

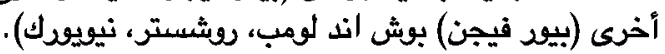

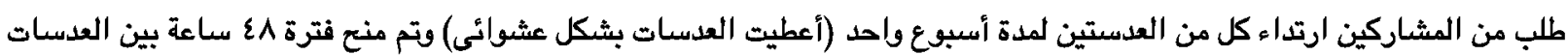

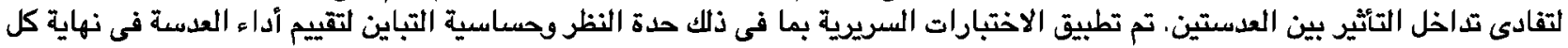

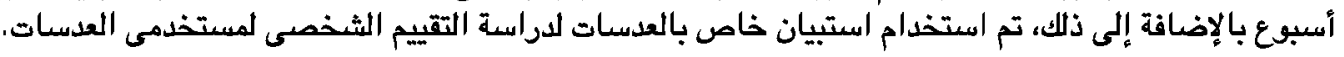

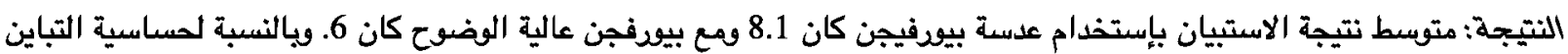

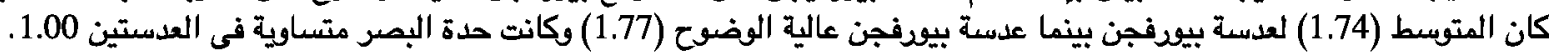

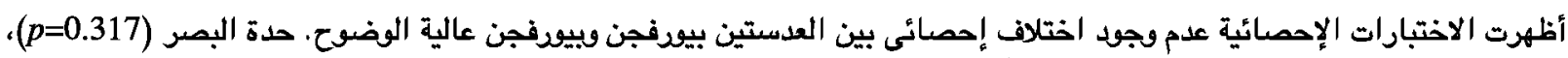

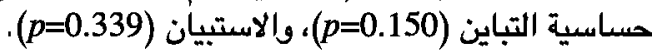

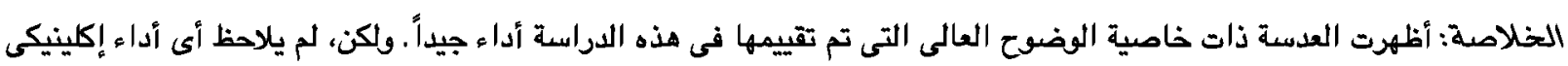
مختف بين العدسات عالية الوضوح والعدسة غير عالية الوضوح على مستوى الرؤية. 